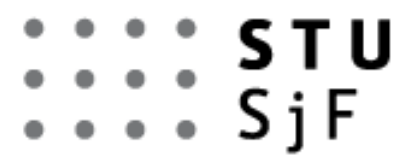

DE GRUYTER OPEN

\title{
FULL FIELD EVALUATION OF THE STRESS TENSOR COMPONENTS IN 2D PHOTOELASTICITY VIA COMPUTER SOFTWARE
}

\author{
PAŠKA Zbyněk ${ }^{1}$, FOJTÍK František ${ }^{1,2}$, FERFECKI Petr ${ }^{1,2}$ \\ ${ }^{1} V \check{B} B$-Technical University of Ostrava, Faculty of Mechanical Engineering, Department of Applied Mechanics, \\ 17.listopadu 15, Ostrava, 17. listopadu 15/2172, 70833 Ostrava-Poruba, Czech Republic; email: \\ zbynek.paska@vsb.cz \\ ${ }^{2}$ VŠB-Technical University of Ostrava, IT4Innovations, 17.listopadu 15, Ostrava, 17. listopadu 15/2172, 708 \\ 33 Ostrava-Poruba, Czech Republic
}

\begin{abstract}
The aim of this work is to find out the components of stress tensor in plane specimens. For this purpose the photoelasticity methodology is used. In order to make this technique more comfortable for use, there was developed an algorithm in MATLAB program. The results are compared with numerical solution. The main advantages of the developed algorithm are the speed and the capabilities to extend to analyze the plastic deformation and strain conditions in the material during forming processes.
\end{abstract}

KEYWORDS: Photoelasticity, isoclinic, isochromatic fringe pattern, Cauchy stress tensor.

\section{Introduction}

The contribution is focusing on issues of $2 \mathrm{D}$ photoelasticity. This technique enables to determination the state of stresses in the transparent specimens, which have a property called birefringence or double refraction. Measurements are performed on a polariscope that creates linearly or circularly polarized light. Photoelasticity is a contactless method and provides data in the full area of the specimen. Photoelasticity is also used in the aerospace industry [1].

Photoelasticity is traditionally divided into three categories: (1) transmission photoelasticity, (2) reflection photoelasticity, which is suited for investigation of stress-strain condition on the surface of real (non-transparent) parts and (3) spatial photoelasticity for stress state investigation inside 3D models. Recently, it has arisen new kind of this technique called digital photoelasticity, which utilize digital image processing methods for photoelastic parameters determination. Investigation of stress condition caused by dynamic loads and a residual stresses is also possible.

\section{Structure of the Evaluation Software}

There are two types of fringes obtained from the measurement. Isoclinic fringes that are used for identification of orientation of the principal stresses. Isochromatic fringes these specify an amount of difference of the principal stresses $\left(\sigma_{1}-\sigma_{2}\right)$ at any point. No other information about the components of the stress tensor is available. The computer procedure can be split into subsequent steps: (1) the identification of the contour of the specimen, (2) the filtration of the measured isoclinic and isochromatic pattern into the smoothed patterns, (3) the combination of isoclinic smoothed patterns, (4) the calculation of the principal stress orientations, (5) the determination of the principal stresses in unloaded contour of the 
specimen, (6) the calculation (using the shear difference method) and display of the components of the stress tensor at an arbitrary point of the loaded specimen.

\section{$3 \quad$ Models and Shear Difference Technique}

The proposed algorithm was tested on the thin annular disc, thin disc subjected to compressive force and beam with a hole subjected to bending force (see Fig. 1).

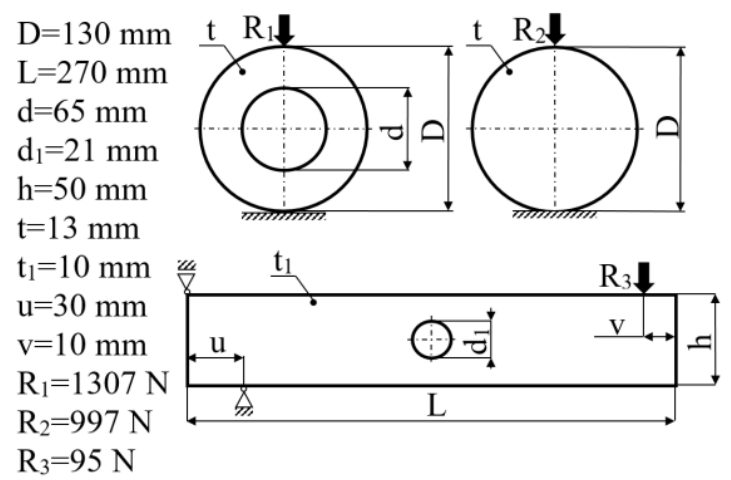

Fig. 1 Investigated specimens.

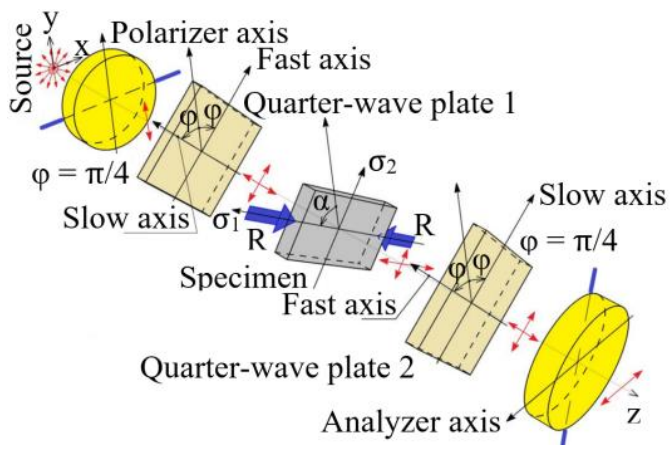

Fig. 2 Scheme of circular polariscope.

A circular polariscope was used for visualizing the isochromatic fringes (see Fig. 2). In this configuration the quarter-wave plates caused that the isoclinic fringes are not present. Isoclinic (Figs. 3, 4) and isochromatic (Figs. 5, 6) fringes can be seen together in one image if the quarter-wave plates are put away.

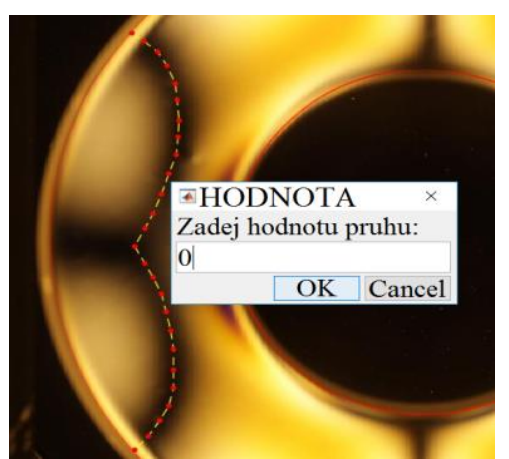

Fig. 3 Rendering of isoclinic $0^{\circ}$.

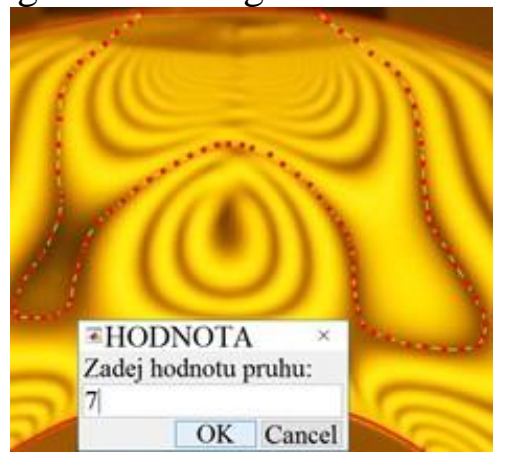

Fig. 5 Rendering of isochromatic fringe order 7 .

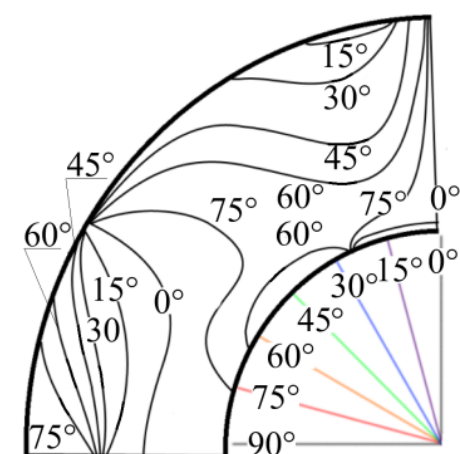

Fig. 4 Isoclinic fringes in one image.

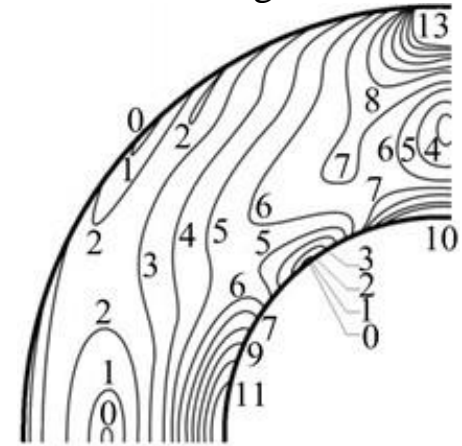

Fig. 6 Isochromatic fringes in one image.

Thin annular disc and thin disc were made from epoxy resin CT 200 (Young's modulus $\mathrm{E}=3124 \mathrm{MPa}$, Poisson's ratio $v=0.35$ ) and beam was made from polycarbonate $(\mathrm{E}=2480 \mathrm{MPa}, v=0.38)$ with stress-fringe value $17.4 \mathrm{~N} \mathrm{~mm}^{-1}$ and $7 \mathrm{~N} \mathrm{~mm}^{-1}$ respectively. 
If it is desired to obtain stress tensor components, there must be implemented some stress separation technique. Shear difference method is used for solution of equilibrium equations expressed in Cartesian coordinates. The body forces are not considered [2]. Inside the model is generated a set of points and for these points is determined the photoelasticity data. Equilibrium equations can be solved numerically and for component $\sigma_{\mathrm{x}}$ is obtained relation

$$
\left(\sigma_{\mathrm{x}}\right)_{j}=\left(\sigma_{\mathrm{x}}\right)_{i}-\sum_{i}^{j}\left(\Delta \tau_{y x} / \Delta y\right) \Delta x ; i=0,1,2 \ldots j,
$$

where $i, j$ are start and end points of integration, $\left(\sigma_{\mathrm{x}}\right)_{\mathrm{i}}$ is stress component in the beginning of integration, $\Delta x$ is the distance between two points $(\Delta y=2 \Delta x)$ and $\Delta \tau_{\mathrm{yx}}$ is the difference of shear stress easily computed from Mohr's circle. The component $\left(\sigma_{\mathrm{y}}\right)_{\mathrm{j}}$ can be determined in a similar way, or it can be expressed from Mohr's circle if the component $\left(\sigma_{\mathrm{x}}\right)_{\mathrm{j}}$ is known.

\section{$4 \quad$ Experimental Results}

After investigation of the entire area of the model or only its part the results are accessible in specially designed software module. It is possible to display plane stress tensor components (Figs. 7, 8), both principal stresses (Fig. 9) and von Misses or Tresca's stress (Fig. 10) there.

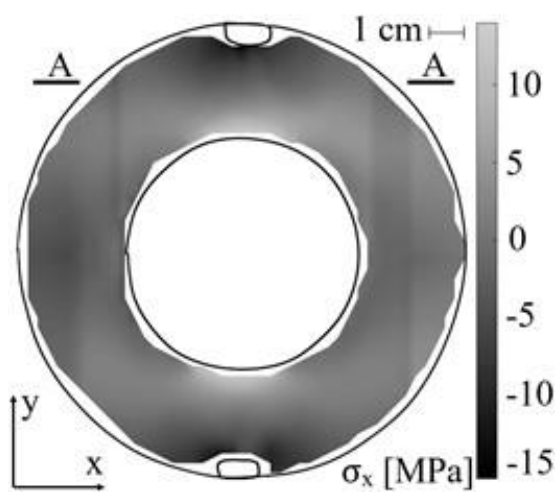

Fig. 7 Stress component $\sigma_{\mathrm{x}}$ in the annular disc.

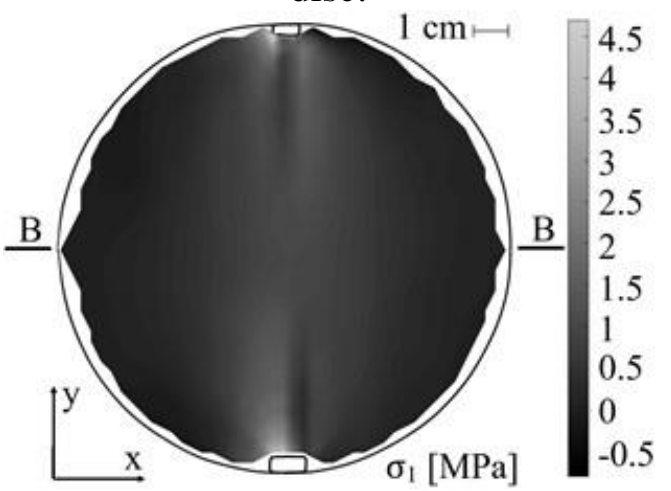

Fig. 9 First principal stress $\sigma_{1}$ in the thin disc.

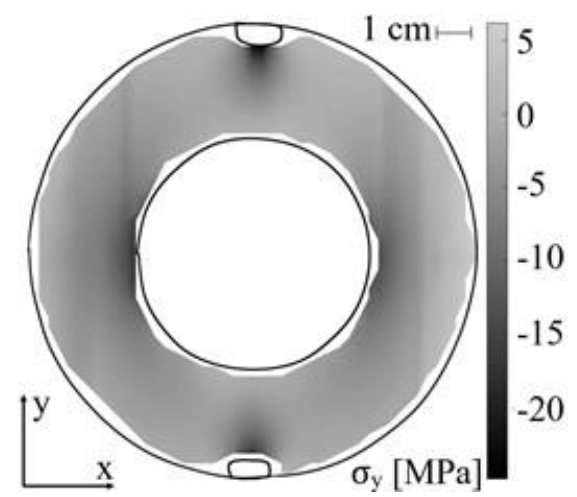

Fig. 8 Stress component $\sigma_{\mathrm{y}}$ in the annular disc.

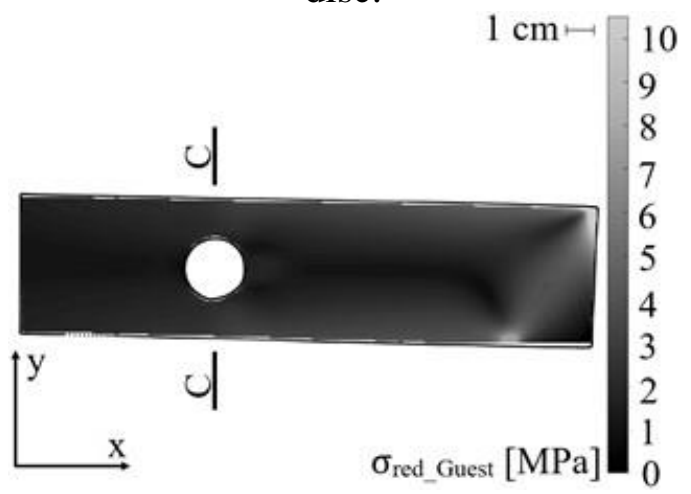

Fig. 10 Maximum shear stresses in the beam.

\section{Comparison with FEM Solution}

Results obtained from experimental (Exp) approach were compared with the numerical solution (FEM) in certain cross-section position on the models. The analytical solution (Ana) was also obtained for the disc. Resultant diagrams are shown in Figs. 11-14. 


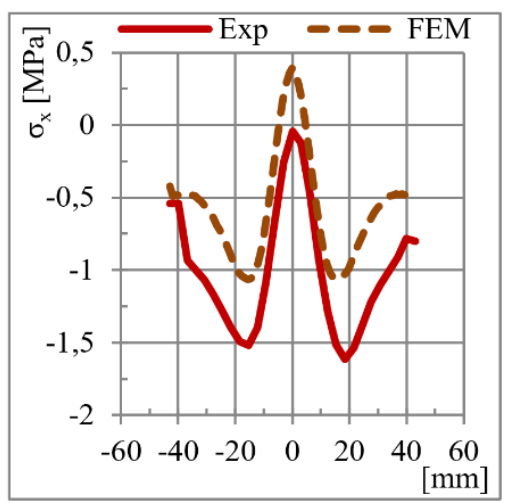

Fig. 11 Distribution of stress $\sigma_{\mathrm{x}}$ in the thin annular disc (cross-section A-A).

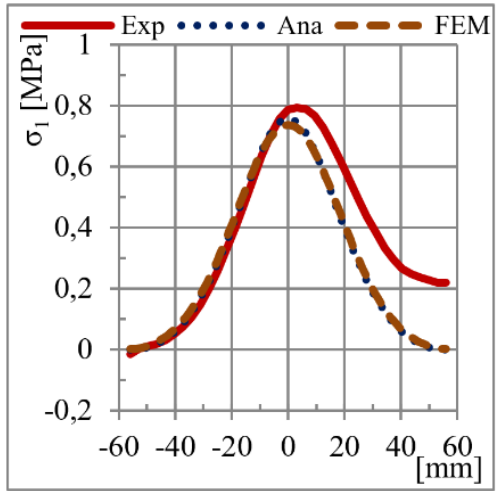

Fig. 13 Distribution of first principal stress in the thin disc (cross-section B-B).

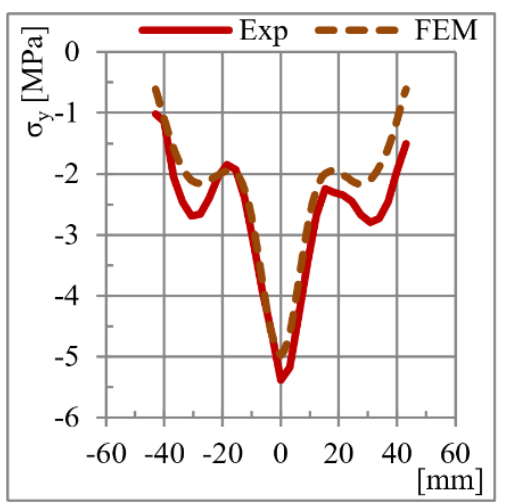

Fig. 12 Distribution of stress $\sigma_{\mathrm{y}}$ in the thin annular disc (cross-section A-A).

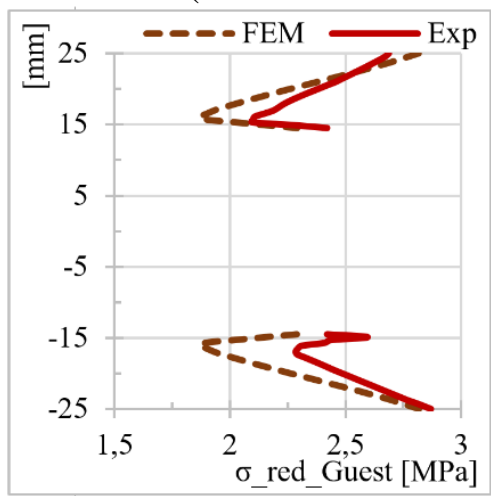

Fig. 14 Distribution of maximum shear stress in thin beam (cross-section C-C).

\section{CONCLUSION}

A computer procedure has been proposed for evaluation of stress tensor components from isochromatic and isoclinic images. The algorithm was tested on three specimens made from optically sensitive materials. The results obtained from experimental solution were compared with results obtained by FEM analysis. The results are considered satisfactory, especially due to accuracy of the input data and the separation technique. The future work will be focused on topics regarding digital photoelasticity and its applicability for solution strain and stress conditions in specimens during forming processes [3].

\section{ACKNOWLEDGEMENT}

This work was supported by The Ministry of Education, Youth and Sports from the National Programme of Sustainability (NPU II) project „IT4Innovations excellence in science - LQ1602“, by the Czech Science Foundation (GA15-18274S) and by the Specific Research (SP2016/145). The support is gratefully acknowledged.

\section{REFERENCES}

[1] K. RAMESH. Digital Photoelasticity. Advanced Techniques and Applications. Berlin, Springer Verlag, 2013. 410 p. 1st ed. ISBN 978-364-2640-995.

[2] A. S. KHAN, X. WANG, Strain Measurements and Stress Analysis, New Jersey, Prentice Hall, 2001. 247 p. ISBN 978-013-0800-763.

[3] PETRUSKA, J., P. MACURA. Pass rolling and material formability - numerical and experimental analysis. Journal of Mechanical Engineering - Strojnicky časopis, 1997(6), 403-414. ISSN 0039-2472 